\title{
Wind Turbine Cross-Sectional Stiffness Analysis Using Internally Layered Solid
} Elements

\author{
Couturier, Philippe; Krenk, Steen
}

Published in:

A I A A Journal

Link to article, DOI:

10.2514/1.J054543

Publication date:

2016

Document Version

Peer reviewed version

Link back to DTU Orbit

Citation (APA):

Couturier, P., \& Krenk, S. (2016). Wind Turbine Cross-Sectional Stiffness Analysis Using Internally Layered Solid Elements. A I A A Journal, 54(7), 2149-2159. https://doi.org/10.2514/1.J054543

\section{General rights}

Copyright and moral rights for the publications made accessible in the public portal are retained by the authors and/or other copyright owners and it is a condition of accessing publications that users recognise and abide by the legal requirements associated with these rights.

- Users may download and print one copy of any publication from the public portal for the purpose of private study or research.

- You may not further distribute the material or use it for any profit-making activity or commercial gain

- You may freely distribute the URL identifying the publication in the public portal

If you believe that this document breaches copyright please contact us providing details, and we will remove access to the work immediately and investigate your claim 


\title{
Wind Turbine Cross-Sectional Stiffness Analysis using Internally Layered Solid Elements
}

\author{
Philippe Couturier ${ }^{1,2}$ and Steen Krenk ${ }^{1}$ \\ 1) Department of Mechanical Engineering \\ Technical University of Denmark, Lyngby, Denmark \\ 2) Siemens Wind Power, Brande, Denmark
}

\begin{abstract}
An efficient finite element modelling approach is presented for analyzing the general cross-section stiffness properties and stress distribution of thin and thick-walled sections with isotropic and general anisotropic materials. The procedure is based on discretizing the walls of the section using a single layer of displacement based elements whereby the element's stiffness is obtained using Gaussian quadrature through each layer. The interlaminar stresses are recovered at points of interest via a $3 \mathrm{D}$ equilibrium-based postprocessing scheme that utilises the distribution of in-plane stress gradients derived in the local laminate coordinate system. The theory is illustrated by application to two composite sections of various shapes and material layups.
\end{abstract}

\section{Introduction}

Rotor blade design in current multi-megawatt wind turbines must adapt to a continually changing market which is driven by the increasing use worldwide of wind energy as a way to diversify the energy supply. To meet the requirements associated with specific installation sites such as power rating and wind conditions, rotor blades are designed using complex crosssection geometries made of composite fiber materials. The modelling approach used must provide accurate predictions of the blade behaviour while being able to easily accommodate drastic geometry and material changes from previous designs.

Given that cross-section dimensions of rotor blades are much smaller than their overall length, beam models can be used to precisely and effectively predict their behaviour. Using beam elements, the complex 3D behaviour of each blade can be modelled using only $10^{2}-10^{3}$ degrees of freedom (DOF) compared to $10^{5}-10^{6}$ DOF when using shell elements. Crosssection properties constitute an essential part of any beam model. As such the cross-section program should be able to accurately model thin-walled parts, lamina built-up parts, and more massive parts while maintaining design flexibility.

Three theories which rely on Finite Element discretization of the cross-section have been shown to provide accurate generalized stiffness properties for wind turbine rotors. The first two approaches are based on advanced kinematic analysis of beams namely the theories developed by Giavotto et al. [1, 2], called nonhomogeneous anisotropic beam section analysis (NABSA), and that of Cesnik and Hodges [3, 4], called variational asymptotic beam sectional 
analysis (VABS). More recently, Couturier et al. [5] developed a cross-sectional analysis procedure which avoids the use of any special 2D theory by analyzing a thin slice of the beam using a single layer of 3D finite elements on which six independent deformation modes corresponding to extension, torsion, homogeneous bending and homogeneous shear are prescribed by imposing suitable displacement increments across the slice.

The accuracy of the generalized stiffness properties as well as the flexibility of modelling using one of these Finite Element based theories is largely based on the discretization approach. The conventional finite element meshing approach is to model each layer in the blade walls using one or more elements through the thickness $[4,6]$. No special kinematic behaviour of the laminate is assumed and the behaviour of individual layers is explicitly taken into considerations. Stresses in each layer can therefore be recovered directly from the constitutive relation. The number of elements will depend on the number of layers which for a typical wind turbine cross-section will be $10^{3}-10^{5}$ elements. This large number of elements requires a significant meshing effort and limits the flexibility in geometry and material layup of an automatic meshing procedure.

Another discretization approach is to model thin-walled parts of the blades using elements with cubic-linear interpolation in the cross-section plane. Only one element is used in the wall thickness and its material properties are taken as the thickness weighted average of the lamina properties $[5,7]$. This enables to model a wind turbine cross-section using less than fifty elements. The averaging of material properties does not capture the effect of the position of each lamina which prevents the calculation of interlaminar stresses and limits its use to thin walled parts.

A preliminary investigation of the use of partial hybrid elements to model cross-sections has been done by Márquez [8]. The method requires the development of new elements which use independent approximations for the in-plane displacements and transverse stress components.

In the present paper, internally layered solid elements previously used to model 3D composite structures $[9,10,11,12]$ are extended to finite-element modelling of thin to thick composite cross-sections. Laminates are modelled using a single element through the wall thickness whereby the element's stiffness is obtained from integration of the stiffness of the individual lamina across the thickness. In-plane stresses are determined directly via the constitutive relation and transverse stresses are recovered via equilibrium equations of 3D elasticity derived in the laminate coordinate system. The internally layered element formulation has been implemented in the cross-sectional analysis program from Couturier et al [5], and this program has been used to provide realistic examples of composite tubular structure and a wind turbine blade cross-section.

The advantage of the present internally layered element modelling approach is that it provides and easy and efficient way of modelling thin and thick walled composite cross-sections using few elements which are based on well-established 3D displacement Finite Element theories. The stress recovery method allows to calculate all stress components in the wall of the structure regardless of the curvature and material layup.

\section{Summary of Cross-Sectional Stiffness Calculation}

This section summarises a method for analysis of the stiffness properties of general crosssections with arbitrary geometry and material distribution that is the basis for the develop- 
ments of this paper. The reader is referred to Couturier et al. [5] for an exhaustive discussion of the formulation.

The method is based on the analysis of a cross-section of a beam which is extruded into a 3D straight prismatic slice of finite thickness $l$. The slice has a coordinate system with its origin located in the center cross-section plane and with longitudinal coordinate $x_{3}$ and crosssection coordinates $x_{1}$ and $x_{2}$, as shown in figure 1(a). The six generalized section forces and moments associated with each cross-section plane along $x_{3}$ are grouped together in the force

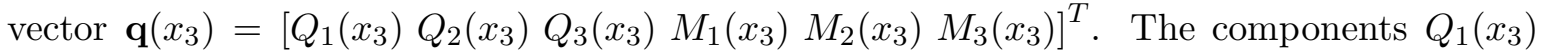
and $Q_{2}\left(x_{3}\right)$ are two shear forces, and $Q_{3}\left(x_{3}\right)$ is the axial force. The components $M_{1}\left(x_{3}\right)$ and $M_{2}\left(x_{3}\right)$ are bending moments, and $M_{3}\left(x_{3}\right)$ is the torsion moment component with respect to the origin of the reference coordinate system. The internal forces and moments components are illustrated in figure $1(\mathrm{~b})$.

In the present paper, the procedure to obtain the cross-section stiffness properties is summarized in two parts. In the first part, the six equilibrium states of the slice are used to obtain a relation between the cross-section stiffness matrix and the slice flexibility matrix. In the second part, a displacement based Finite Element approach is used to obtain the slice flexibility matrix.

\subsection{Cross-Section Flexibility}

The statics of the slice can be described by six equilibrium modes, namely the homogeneous states of extension, torsion, bending, and shear. From equilibrium consideration the slice without external loads will exhibit constant internal normal force, shear forces and torsion moment, while the bending moments will vary linearly with the shear force as gradient. The six equi-

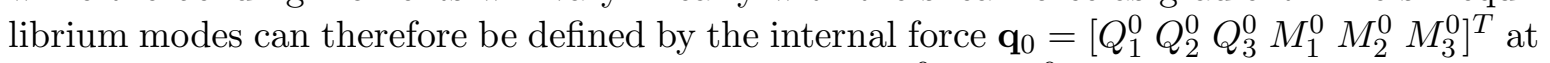
the center of the slice. Since the constant shear forces $Q_{1}^{0}$ and $Q_{2}^{0}$ lead to linear anti-symmetric moment variation, the distribution of internal forces in the slice in terms of their values $\mathbf{q}_{0}$ at the center cross-section of the slice follows as

$$
\left[\begin{array}{l}
Q_{1}\left(x_{3}\right) \\
Q_{2}\left(x_{3}\right) \\
Q_{3}\left(x_{3}\right) \\
M_{1}\left(x_{3}\right) \\
M_{2}\left(x_{3}\right) \\
M_{3}\left(x_{3}\right)
\end{array}\right]=\left[\begin{array}{cccccc}
1 & 0 & 0 & 0 & 0 & 0 \\
0 & 1 & 0 & 0 & 0 & 0 \\
0 & 0 & 1 & 0 & 0 & 0 \\
0 & x_{3} & 0 & 1 & 0 & 0 \\
-x_{3} & 0 & 0 & 0 & 1 & 0 \\
0 & 0 & 0 & 0 & 0 & 1
\end{array}\right]\left[\begin{array}{c}
Q_{1}^{0} \\
Q_{2}^{0} \\
Q_{3}^{0} \\
M_{1}^{0} \\
M_{2}^{0} \\
M_{3}^{0}
\end{array}\right]
$$

or in a more compact notation

$$
\mathbf{q}\left(x_{3}\right)=\mathbf{T}\left(x_{3}\right) \mathbf{q}_{0},
$$

where the interpolation matrix $\mathbf{T}\left(x_{3}\right)$ is defined in (1).

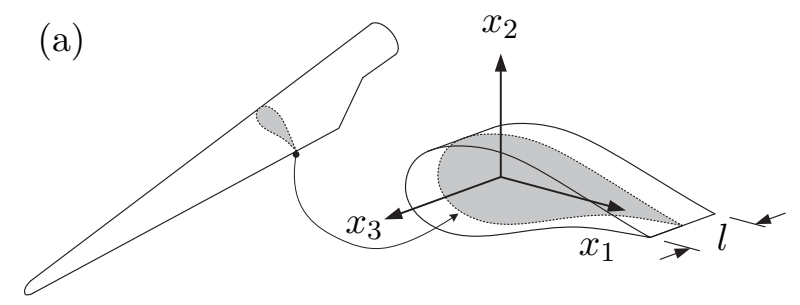

(b)

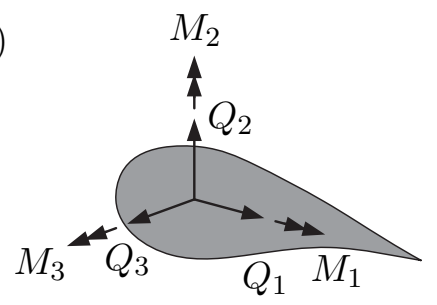

Figure 1: (a) Slice coordinate system (b) Section forces and moments. 
Following the general formulation of equilibrium based beam elements in Krenk [13], the deformation associated with the internal forces and moments $\mathbf{q}$ is described in terms of six generalized strains, defined by the strain vector $\gamma=\left[\begin{array}{llllll}\varepsilon_{1} & \varepsilon_{2} & \varepsilon_{3} & \kappa_{1} & \kappa_{2} & \kappa_{3}\end{array}\right]^{T}$. The components $\varepsilon_{1}$ and $\varepsilon_{2}$ are generalized shear strains, and $\varepsilon_{3}$ is the axial strain. Similarly $\kappa_{1}$ and $\kappa_{2}$ are the components of bending curvature, while $\kappa_{3}$ is the rate of twist. The generalized strain $\gamma$ is defined such that it is conjugate to the internal force vector $\mathbf{q}$ with respect to energy. Thus, the specific elastic energy associated with a cross-section can be given either as a product of the generalized strain $\gamma$ and internal force vector $\mathbf{q}$ or using the cross-section flexibility matrix $\mathbf{C}$ of the linear elastic slice

$$
W_{s}\left(x_{3}\right)=\frac{1}{2} \gamma\left(x_{3}\right)^{T} \mathbf{q}\left(x_{3}\right)=\frac{1}{2} \mathbf{q}\left(x_{3}\right)^{T} \mathbf{C} \mathbf{q}\left(x_{3}\right),
$$

The inverse of the flexibility matrix $\mathbf{C}$ is the six by six cross-section stiffness matrix $\mathbf{D}$. Both matrices can contain up to 21 independent entries where the off-diagonal entries represent geometrical or material induced couplings. From the constant properties in the longitudinal direction $x_{3}$, the flexibility matrix of the slice's equilibrium modes follows from integration of the cross-section flexibility relation (3) over the slice length

$$
W_{e}=\int_{-l / 2}^{l / 2} W_{s}\left(x_{3}\right) d x_{3}=\frac{1}{2} \int_{-l / 2}^{l / 2} \mathbf{q}\left(x_{3}\right)^{T} \mathbf{C} \mathbf{q}\left(x_{3}\right) d x_{3} .
$$

Representing the internal forces and moments via the mid-point values $\mathbf{q}_{0}$ by (2) yields

$$
W_{e}=\frac{1}{2} \mathbf{q}_{0}^{T} \mathbf{H} \mathbf{q}_{0} .
$$

where the slice flexibility matrix $\mathbf{H}$ corresponding to the six equilibrium modes is defined by the integral

$$
\mathbf{H}=\int_{-l / 2}^{l / 2} \mathbf{T}\left(x_{3}\right)^{T} \mathbf{C} \mathbf{T}\left(x_{3}\right) d x_{3} .
$$

Carrying out the integration in explicit form provides the relation between the slice flexibility matrix $\mathbf{H}$ and the cross-section flexibility matrix $\mathbf{C}$

$$
\mathbf{H}=l\left[\begin{array}{cccccc}
C_{11}+\frac{l^{2}}{12} C_{55} & C_{12}-\frac{l^{2}}{12} C_{54} & C_{13} & C_{14} & C_{15} & C_{16} \\
C_{21}-\frac{l^{2}}{12} C_{45} & C_{22}+\frac{l^{2}}{12} C_{44} & C_{23} & C_{24} & C_{25} & C_{26} \\
C_{31} & C_{32} & C_{33} & C_{34} & C_{35} & C_{36} \\
C_{41} & C_{42} & C_{43} & C_{44} & C_{45} & C_{46} \\
C_{51} & C_{52} & C_{53} & C_{54} & C_{55} & C_{56} \\
C_{61} & C_{62} & C_{63} & C_{64} & C_{65} & C_{66}
\end{array}\right] .
$$

It is seen how the slice flexibility matrix is a function of the cross-section properties as well as the thickness $l$ of the slice. It is observed that for a finite-thickness slice the bending flexibility parameters $C_{55}, C_{44}, C_{54}$, and $C_{54}$ of the cross-section contribute to the constant-shear modes of the beam due to their linearly varying bending moment. If the flexibility matrix of a slice with arbitrary length is know, the relation in (7) can be used to recover the representative cross-section stiffness properties. 
(a)

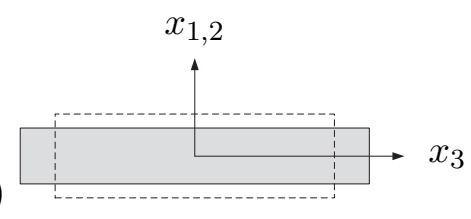

(c)

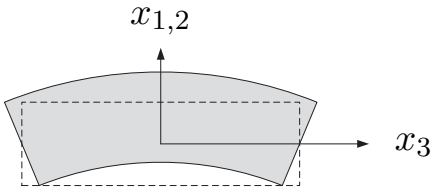

(b)

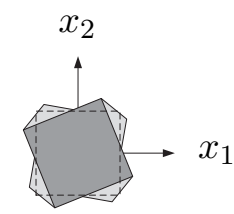

(d)

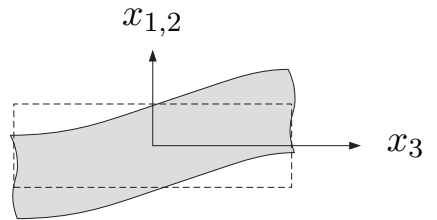

Figure 2: Deformation modes: (a) Extension, (b) Twist, (c) Bending, (d) Shear.

\section{$2.2 \quad$ Finite Element Representation}

In the present methodology, the slice flexibility matrix $\mathbf{H}$ is obtained using the Finite Element Method whereby the slice is discretized using 3D isoparametric finite elements. The use of 3D finite elements enables a simple and direct representation of material discontinuities and general anisotropy. The model needs to capture the third order displacement field in the axial direction $x_{3}$ associated with prismatic beams. This displacement field is captured using a single layer of element in the thickness direction with lengthwise Hermitian interpolation. The displacement field in vector components $\mathbf{u}\left(x_{j}\right)=\left[u_{j}\right]^{T}$ where the $j$ index ranges from one to three are described using finite elements as

$$
\mathbf{u}\left(x_{j}\right)=\mathbf{N}\left(x_{j}\right) \mathbf{v},
$$

where $\mathbf{N}\left(x_{j}\right)$ are the shape functions corresponding to the nodal displacement which are contained in the column vector $\mathbf{v}=\left[\mathbf{v}_{1}, \ldots, \mathbf{v}_{m}\right]^{T}$ where $m$ is the total number of nodes. The nodal degrees of freedom are defined as $\mathbf{v}_{i}=\left[u_{\alpha}, u_{3}, u_{\alpha}^{\prime}, u_{3}^{\prime}\right]^{T}$. The components $u_{\alpha}$ and $u_{3}$ represent the in-plane and axial displacements, respectively, while the components $u_{\alpha}^{\prime}$ and $u_{3}^{\prime}$ represent the corresponding derivatives with respect to the axial coordinate $x_{3}$. The static component vector conjugate to the displacement vector $\mathbf{v}_{i}$ is the force vector $\mathbf{p}_{i}=\left[f_{\alpha}, f_{3}, f_{\alpha}^{\prime}, f_{3}^{\prime}\right]^{T}$. The components $f_{\alpha}$ and $f_{3}$ represent the three force components, while the components $f_{\alpha}^{\prime}$ and $f_{3}^{\prime}$ represent three moments components conjugate to the displacement derivatives $u_{\alpha}^{\prime}$ and $u_{3}^{\prime}$, respectively.

The procedure consists in solving the finite element problem corresponding to six independent equilibrium states. The six independent equilibrium states are chosen as the deformation modes corresponding to extension, twist, bending, and shear. Each of the states are obtained by imposing appropriate displacements on the end-sections of the beam slice. The deformation modes are illustrated in figure 2 for the case of a square orthotropic cross-section where the undeformed slice is sketched using dotted lines. The generalized nodal displacements, conjugate generalized nodal forces, and mid-section-force component vector of the six equilibrium states $j=1, \ldots, 6$ are contained in the column vectors $\tilde{\mathbf{v}}_{j}, \tilde{\mathbf{p}}_{j}$ and $\tilde{\mathbf{q}}_{0 j}$. The vectors are grouped in the following three matrices

$$
\mathbf{V}=\left[\tilde{\mathbf{v}}_{1}, \ldots, \tilde{\mathbf{v}}_{6}\right], \quad \mathbf{P}=\left[\tilde{\mathbf{p}}_{1}, \ldots, \tilde{\mathbf{p}}_{6}\right], \quad \mathbf{R}=\left[\tilde{\mathbf{q}}_{01}, \ldots, \tilde{\mathbf{q}}_{06}\right]
$$

A general equilibrium state can now be represented as a linear combination of the six basic equilibrium states introduced above. The components of this representation are denoted 
$\mathbf{s}=\left[s_{1}, \cdots, s_{6}\right]^{T}$, whereby the nodal displacements, nodal forces and section-forces of the center section take the form

$$
\mathbf{v}=\sum_{j=1}^{6} \tilde{\mathbf{v}}_{j} s_{j}=\mathbf{V} \mathbf{s}, \quad \mathbf{p}=\sum_{j=1}^{6} \tilde{\mathbf{p}}_{j} s_{j}=\mathbf{P} \mathbf{s}, \quad \mathbf{q}_{0}=\sum_{j=1}^{6} \tilde{\mathbf{q}}_{0 j} s_{j}=\mathbf{R} \mathbf{s} .
$$

The elastic energy of the slice can now be expressed alternatively in terms of the flexibility matrix $\mathbf{H}$ either by use of (5), or as the product of the nodal forces $\mathbf{P}$ and displacements $\mathbf{V}$ as given by the representations (10),

$$
W_{e}=\frac{1}{2} \mathbf{s}^{T} \mathbf{R}^{T} \mathbf{H} \mathbf{R} \mathbf{s}=\frac{1}{2} \mathbf{s}^{T} \mathbf{V}^{T} \mathbf{P} \mathbf{s} .
$$

This is an identity valid for arbitrary component vectors $\mathbf{s}$ and noting that the matrix $\mathbf{R}$ is regular, the flexibility matrix $\mathbf{H}$ follows as

$$
\mathbf{H}=\mathbf{R}^{-T}\left(\mathbf{V}^{T} \mathbf{P}\right) \mathbf{R}^{-1} .
$$

This procedure determines the 36 elements of the flexibility matrix $\mathbf{H}$ from the six load-cases solved by the finite element analysis of the 3D slice. The cross-section flexibility matrix $\mathbf{C}$ can then be calculated using the finite-length flexibility relation (7), and the corresponding cross-section stiffness matrix $\mathbf{D}$ is obtained by inversion of this flexibility matrix. The present cross-section analysis method has been implemented and validated in a computer program called CrossFlex (Cross-section Flexibility) $[15,14,5]$.

\section{Internally Layered Element}

The discretization of the slice needed for the finite element analysis presented in the previous section is general in the sense that it can accommodate displacement based elements with any shape, any interpolation function, and any anisotropic material distribution in the crosssection plane. Using this flexibility of the method, a meshing approach which requires very few elements can be developed using elements which can each capture the behaviour of several different material layers.

Consider a hexahedron finite element described in therms of the intrinsic coordinates $\boldsymbol{\xi}=[\xi, \eta, \zeta]^{T}$ where the coordinate $\zeta$ is collinear with the global axial coordinate. The intrinsic coordinates cover the range $-1 \leq \xi, \eta, \zeta \leq 1$. The element stiffness matrix is then given $[16,17]$ by

$$
\mathbf{K}_{e}=\int_{-1}^{1} \int_{-1}^{1} \int_{-1}^{1} \mathbf{B}^{T} \mathbf{E} \mathbf{B} J \mathrm{~d} \xi \mathrm{d} \eta \mathrm{d} \zeta
$$

where $\mathbf{E}$ is the material constitutive matrix, $\mathbf{B}$ is the strain-displacement matrix, and $J$ is the determinant of the Jacobian matrix. The equation (13) is evaluated using Gaussian quadrature.

Consider now that the element of thickness $t$ contains $n$ layers of different material as shown in figure 3. Note that in the current formulation, $\eta$ is chosen to be perpendicular to the layer plane. Care must be taken to ensure proper orientation of the elements in the mesh. The material constitutive matrix and thickness of the $k^{\prime}$ th layer is defined as $\mathbf{E}_{k}$ and $h_{k}$, respectively. In order to use to same Gauss point and weight factor values, the limits 
of integration should remain from -1 to 1 . This is achieved using the following coordinate transformation [10] where $\eta$ is replaced by $\eta_{k}$ which ranges from -1 to 1 in each layer

$$
\eta=-1+\frac{1}{t}\left[-h_{k}\left(1-\eta_{k}\right)+2 \sum_{j=1}^{k} h_{j}\right] .
$$

from which it follows that

$$
\mathrm{d} \eta=\frac{h_{k}}{t} \mathrm{~d} \eta_{k} .
$$

Carrying out the numerical integration over each layer by substitution of (14) and (15) in (13) yields

$$
\mathbf{K}_{e}=\sum_{k=1}^{n} \int_{-1}^{1} \int_{-1}^{1} \int_{-1}^{1} \mathbf{B}^{T} \mathbf{E}_{k} \mathbf{B} J \frac{h_{k}}{t} \mathrm{~d} \xi \mathrm{d} \eta_{k} \mathrm{~d} \zeta .
$$

It is clear from this expression that the number of Gauss integration points in proportional to the number of layers. Moreover, if the element contains only one material layer the expression for a 3D solid element is recovered. This numerical integration through the laminate is also used in plate and shell theories [18]. Although out of the scope of this paper, the concept of internally layered elements can also be used to determine the general cross-section mass properties. The required analysis is simpler than the one to obtain the stiffness properties as only the density of each layer needs to be integrated over the element.

\section{Stress Recovery}

Layered composites have continuous in-plane strain components across lamina interfaces and depending on the lamina material properties may have discontinuous interlaminar strain components across lamina interfaces. From the displacement continuity inside the element, internally layered elements with appropriate interpolation functions are able to capture the in-plane strain distribution and consequently the in-plane stress distribution can be obtained from the constitutive relations. Internally layered elements are however enable to capture the interlaminar strain discontinuity which prevents calculation of the interlaminar stress distribution directly from constitutive relations.

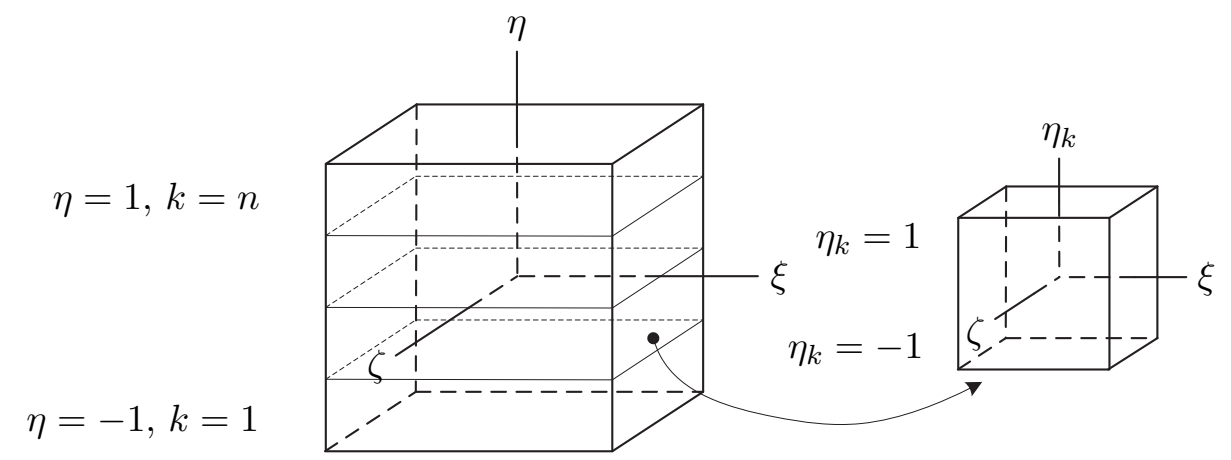

Figure 3: Internally layered element intrinsic $\{\boldsymbol{\xi}, \boldsymbol{\eta}, \boldsymbol{\zeta}\}$ coordinate system and layer $\left\{\boldsymbol{\xi}, \boldsymbol{\eta}_{\boldsymbol{k}}, \boldsymbol{\zeta}\right\}$ coordinate system. 


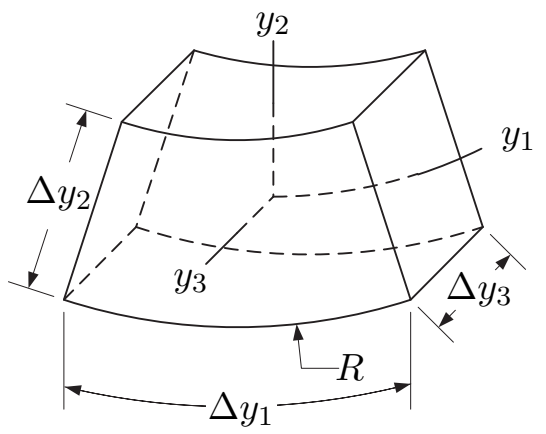

Figure 4: Differential element of the laminate showing the laminate coordinate system.

The importance of the representation of the interlaminar stresses depends on the goal of the analysis. For example, the cross-sectional stiffness analysis is governed by the in-plane stress distribution where the axial stresses dominate the extension and bending stiffnesses and the in-plane shear stresses dominate the torsion and shear stiffnesses. Conversely, delamination of laminates is governed by the interlaminar stress distribution.

A direct way to obtain the transverse shear stress distribution is to consider a differential element inside the laminate with a local laminate coordinate system $\left\{y_{1}, y_{2}, y_{3}\right\}$ as shown in figure 4 where the coordinate $y_{3}$ is collinear with the slice longitudinal axis $x_{3}$, the coordinate $y_{2}$ is in the laminate thickness direction, and the $y_{1}$ axis follows the local laminate curvature defined by the radius of curvature $R$. Adding the surface forces in the three coordinate directions gives the following three equilibrium equations in terms of the stress components in the laminate coordinate system

$$
\begin{gathered}
\frac{\partial \tau_{13}}{\partial y_{1}}+\frac{\partial \tau_{23}}{\partial y_{2}}+\frac{\partial \sigma_{3}}{\partial y_{3}}-\frac{\tau_{23}}{R}=0, \\
\frac{\partial \sigma_{1}}{\partial y_{1}}+\frac{\partial \tau_{12}}{\partial y_{2}}+\frac{\partial \tau_{13}}{\partial y_{3}}-\frac{2 \tau_{12}}{R}=0, \\
\frac{\partial \tau_{12}}{\partial y_{1}}+\frac{\partial \sigma_{2}}{\partial y_{2}}+\frac{\partial \tau_{23}}{\partial y_{3}}+\frac{\sigma_{1}-\sigma_{2}}{R}=0 .
\end{gathered}
$$

Note that when the curvature of the laminate approaches zero, i.e. the radius of curvature $R$ approaches infinity, (17)-(19) reduce to the standard equilibrium equations of 3D elasticity in Cartesian coordinate. The interlaminar stresses $\tau_{23}, \tau_{12}$, and $\sigma_{2}$ can be obtained by integrating (17), (18) and (19) over the thickness of the element. With this approach only the in-plane stresses need to be computed from constitutive relations based on the solution from the finite element analysis.

The distribution of in-plane stress gradients in an element needed in (17)-(19) is obtained by interpolating the in-plane stress gradients in each $y_{1}-y_{3}$ plane containing Gauss points by defining the in-plane stress vector field $\boldsymbol{\sigma}\left(y_{1}, y_{3}\right)=\left[\sigma_{1}, \sigma_{3}, \tau_{13}\right]^{T}$ in each $y_{1}-y_{3}$ plane containing Gauss points using the representation

$$
\boldsymbol{\sigma}\left(y_{1}, y_{3}\right)=\mathbf{N}(\xi, \zeta) \phi
$$

The matrix $\mathbf{N}(\xi, \zeta)$ contains the interpolation functions corresponding to the Gauss point stresses contained in the column vector $\phi=\left[\phi_{1}, \ldots, \phi_{n}\right]^{T}$ where $n$ is the number of Gauss 
points. The three in-plane stresses at each Gauss point are defined as $\boldsymbol{\phi}_{i}=\left[\sigma_{1}, \sigma_{3}, \tau_{13}\right]_{i}^{T}$. An illustration of the stress mapping between the intrinsic coordinate system and the plane in the laminate coordinate system is shown in figure 5 .

The in-plane stress gradients with respect to $y_{1}$ and $y_{3}$ follow directly from differentiation of (20) by ensuring that the $y_{1}$ and $y_{3}$ axis are orthogonal

$$
\begin{gathered}
\boldsymbol{\sigma}_{, y_{1}}\left(y_{1}, y_{3}\right)=\frac{d \xi}{d y_{1}} \mathbf{N}_{, \xi}(\xi, \zeta) \phi=\frac{2}{\Delta s_{1}} \mathbf{N}_{, \xi}(\xi, \zeta) \boldsymbol{\phi} \\
\boldsymbol{\sigma}_{, y_{3}}\left(y_{1}, y_{3}\right)=\frac{d \zeta}{d y_{3}} \mathbf{N}_{, \zeta}(\xi, \zeta) \boldsymbol{\phi}=\frac{2}{l} \mathbf{N}_{, \zeta}(\xi, \zeta) \boldsymbol{\phi} .
\end{gathered}
$$

where,$\varphi$ denotes differentiation with respect to $\varphi$. Note that the scale factors mapping $\xi$ to $y_{1}$ and $\zeta$ to $y_{3}$ have been replaced by the ratio of the plane dimensions in the $\xi-\zeta$ space being two by two to its dimensions in the $y_{1}-y_{3}$ space being $\Delta s_{1}$ by $l$ as shown in figure 5. The arc length $\Delta s_{1}$ can be obtained by fitting a polynomial curve through the Gauss points. Obtaining the in-plane stress gradients in this way avoids the need to transform stress gradients from the global coordinate system to the laminate coordinate system.

Using a piecewise interpolation of the in-plane stress gradients in each $k$ 'th layer in the thickness direction $y_{2}$, the interlaminar stresses are obtained by integrating (17)-(19) by assuming traction free surface on the bottom surface $y_{2}=-t / 2$. This integration through the element thickness guaranties interlaminar stress continuity between the layers and at least one stress free surface.

\section{Applications}

The present section contains an assessment of the effectiveness of using internally layered elements to calculate cross-section stiffness properties and stress distribution of thin and thick walled composite structures. Two applications are considered, namely a composite tube section with soft and stiff core and a realistic wind turbine cross-section.

\subsection{Composite Circular Tube Cross-Section}

This example concerns the circular section with outer radius of $R=0.5 \mathrm{~m}$ shown in figure 6 . The cross-section is build-up as a symmetric sandwich structure where the core occupies $75 \%$

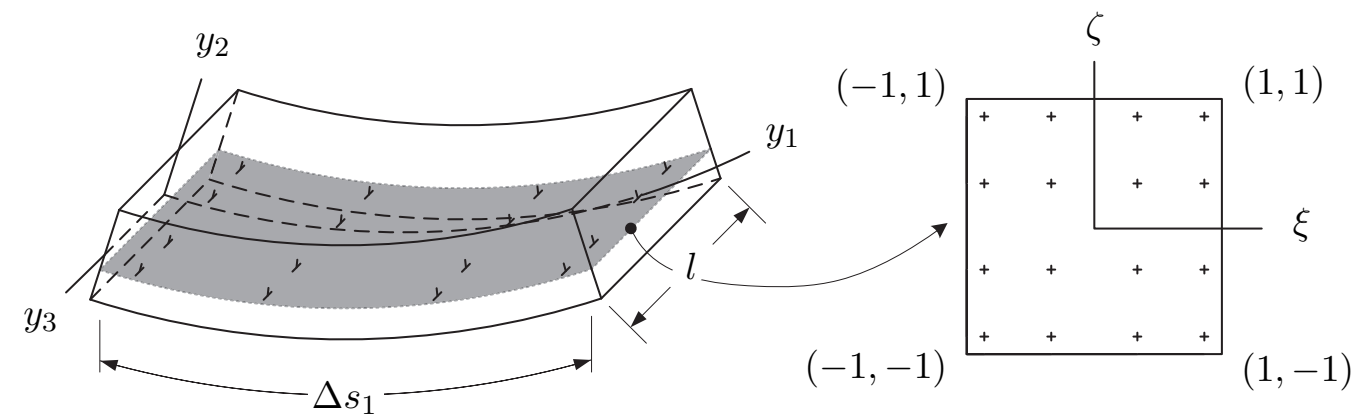

Figure 5: Mapping of in-plane stresses at Gauss points between intrinsic coordinate system and laminate coordinate system. 


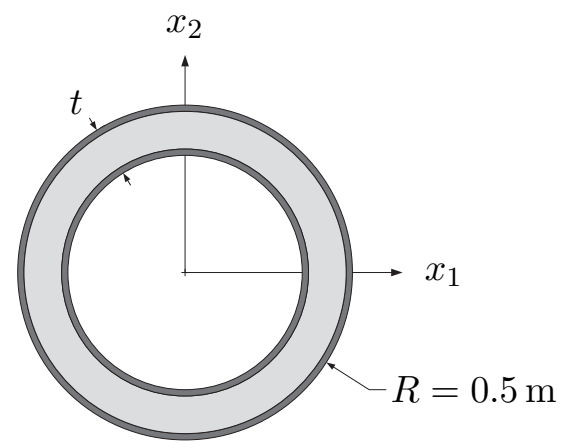

Figure 6: Schematic of circular section with $\boldsymbol{t} / \boldsymbol{R}=\mathbf{0 . 3}$.

of the total wall thickness and the remaining $25 \%$ is divided equally between the two faces. The faces are made of fiberglass-epoxy with the main fiber direction collinear with the beam axis. Two core materials are considered, namely fiberglass-epoxy with the main fiber direction in the circumferential direction and balsa. The material properties are taken from Griffin [19] and Couturier et al. [5] and are listed for reference in Table 1.

The cross-section is analyzed using four models each with a different discretization and material representation combination. Model A, shown in figure 7(a), represents the conventional highly discretized meshing approach where in the thickness direction each fiberglass-epoxy faces are discretized using two elements and the core is discretized using six elements. In the circumferential direction 16 segments are used for a total of 160 solid elements and 8064 DOF. The elements have quadratic interpolation in the cross-section plane and Hermitian cubic interpolation in the axial direction. Model B, shown in figure 7(b), uses a single internally layered element with quadratic shape functions over the thickness and 16 segments

Table 1: Material properties.

\begin{tabular}{lccccc}
\hline Material & $E_{11}[\mathrm{GPa}]$ & $E_{22}=E_{33}[\mathrm{GPa}]$ & $G_{12}=G_{13}[\mathrm{GPa}]$ & $G_{23}[\mathrm{GPa}]$ & $\nu_{12}=\nu_{13}=\nu_{23}$ \\
\hline Triaxial fabric & 24.2 & 8.97 & 4.97 & 3.23 & 0.39 \\
Balsa & 2.07 & 2.07 & 0.14 & 0.863 & 0.22 \\
\hline
\end{tabular}

(a)

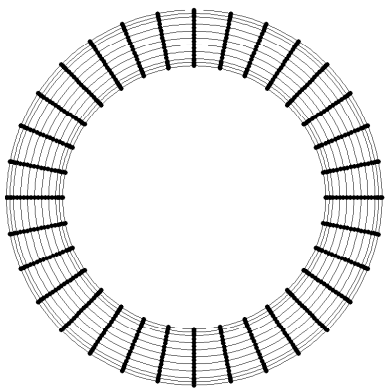

(b)

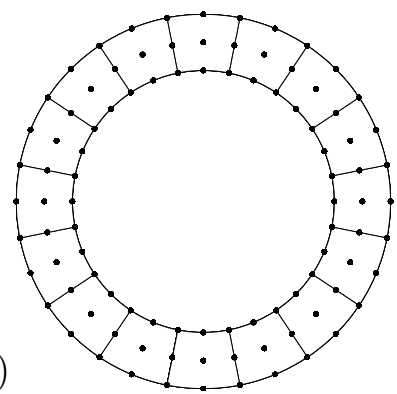

(c)

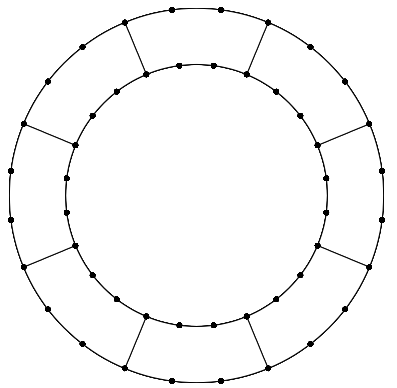

Figure 7: (a) Model A: 160 solid quadratic elements (b) Model B: 16 solid interanlly layered quadratic elements (c) Model C: 8 solid internally layered linear-cubic elements. 

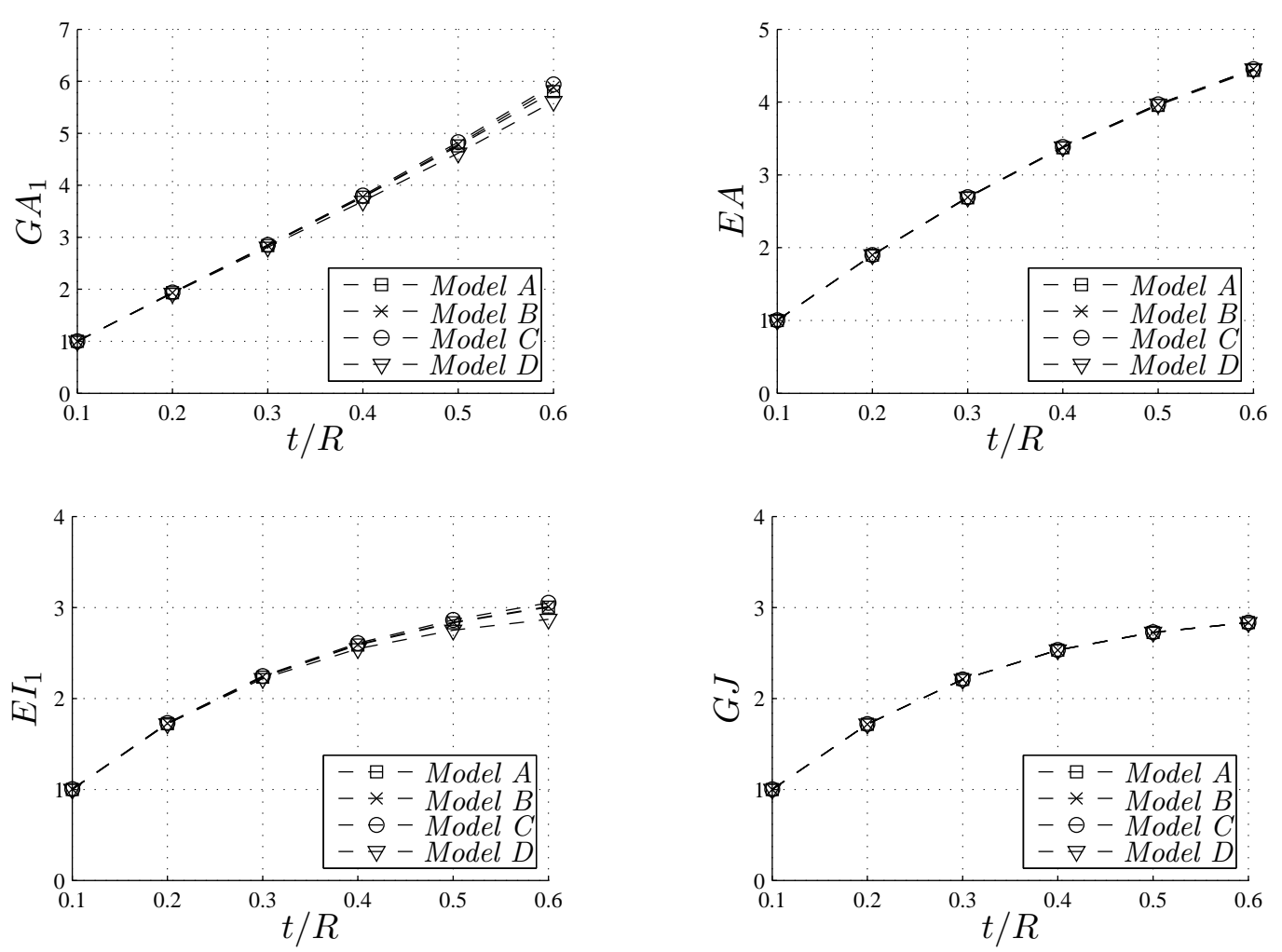

Figure 8: Stiffness properties for fiberglass-epoxy core section normalized with the value obtained using 160 solid elements (Model A) at $\boldsymbol{t} / \boldsymbol{R}=\mathbf{0 . 1}$.

in the circumferential direction for a total of 16 elements and 1152 DOF. Model C, shown in figure $7(\mathrm{c})$, uses internally layered elements with linear-cubic interpolation with a single element in the thickness direction and eight segments in the circumferential direction for a total of eight elements and 576 DOF. Model D also uses eight elements with linear-cubic interpolation however it uses a thickness-weighted average constitutive relations.

The effect of the wall thickness on the cross-section stiffness coefficients calculated using the four models for the case of the fiberglass-epoxy core is shown in figure 8 . The wall thickness was varied from $t / R=1 / 10$ to $t / R=3 / 5$. The reference axis being at the center, only the diagonal terms in the stiffness matrix are non-zero. The diagonal terms are $G A_{1}$, $G A_{2}, E A, E I_{1}, E I_{2}$, and $G J$ representing the shear stiffness about both in-plane axes, the extension stiffness, the bending stiffness about both in-plane axes, and the torsion stiffness, respectively. From symmetry of the section $G A_{1}=G A_{2}$ and $E I_{1}=E I_{2}$.

Stiffness properties obtained using internally layered elements with either linear-cubic (Model C) or quadratic interpolation (Model B) are in good agreement with those obtained using the detailed model (Model A) with a maximum error in bending and shear at $t / R=0.6$ of $1.6 \%$ and $2.4 \%$, respectively. Although comparable results are obtained, models using internally layered elements (Model B and C) required substantially less meshing and computational effort given they need an order of magnitude less elements and only require information about the structure's geometry for node positioning.

It can be seen in figure 8 that for $t / R \leq 0.3$ results obtained using the averaged constitutive 

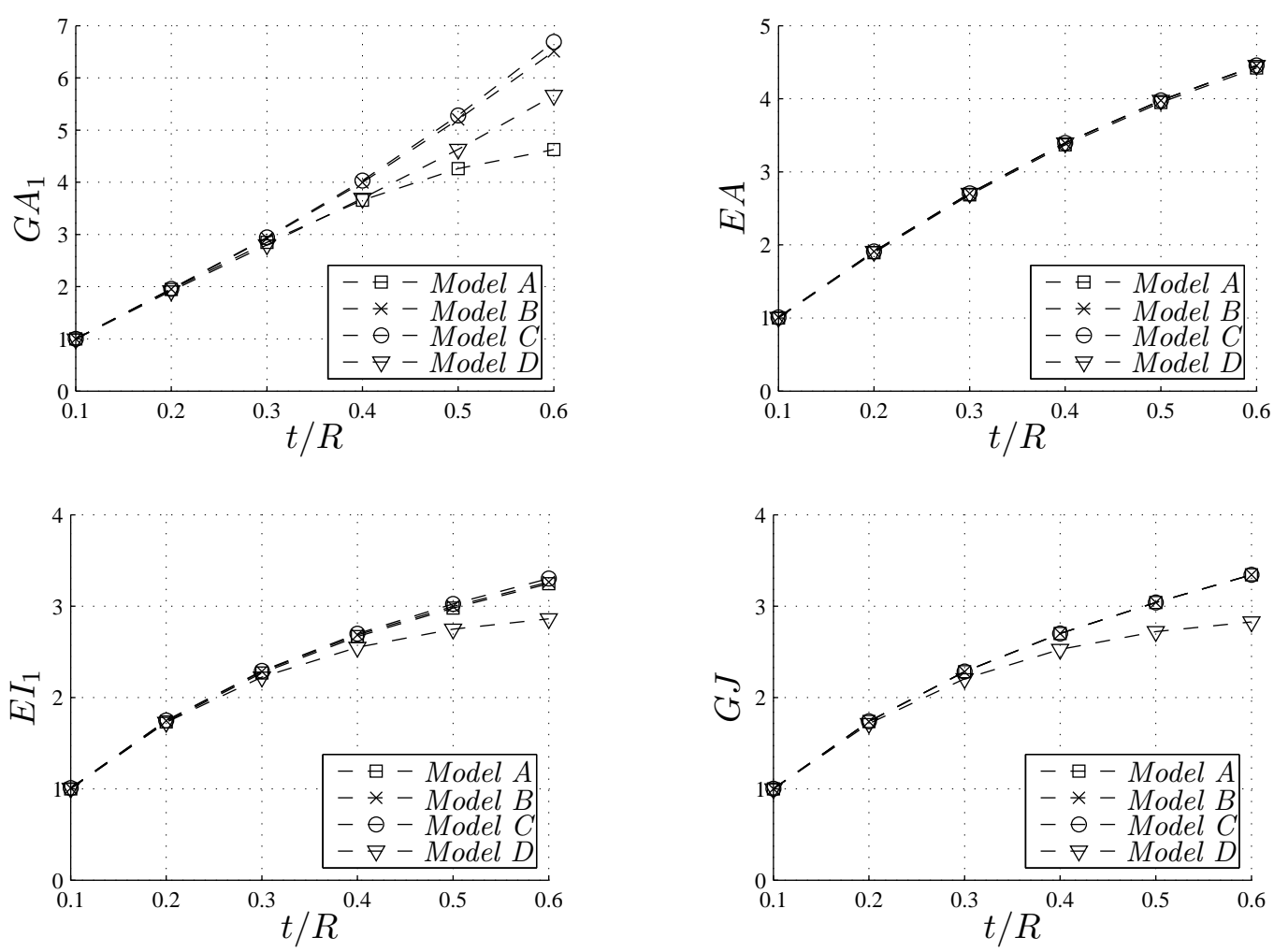

Figure 9: Stiffness properties for balsa core section normalized with the value obtained using 160 solid elements (Model A) at $\boldsymbol{t} / \boldsymbol{R}=\mathbf{0 . 1}$.

relations (Model D) agree well with those obtained with the other methods. As the thickness is further increased it underpredicts the bending and torsional stiffnesses with a maximum error in bending and torsion for the balsa core at $t / R=0.6$ of $12 \%$ and $15 \%$, respectively. This error stems from the nature of the averaging process where the effect of the position of the stiff faces with respect to the neutral axis and the center of twist is lost.

The effect of the wall thickness on the cross-section stiffness coefficients calculated using the four models for the case of the balsa core is shown in figure 9. Sections modelled using internally layered elements with either linear-cubic (Model C) or quadratic interpolation (Model B) are in good agreement with those obtained using the detailed model (Model A) for the extension, bending and torsion stiffnesses for the range of thicknesses studied with a maximum error in bending at $t / R=0.6$ of $1.8 \%$. Shear stiffness obtained using internally layered elements showed a deviation larger than $5 \%$ for wall thicknesses larger than $t / R=0.3$. The reason for this discrepancy is that as the soft balsa core becomes thicker the structure should behave more like two independent concentric tubes. The kinematics of this effect is not captured with the use of single element through the thickness whereby the structure behaves more as two rigidly connected tubes which has a larger stiffness [20]. One should therefore be aware for parts of structures that become increasingly independent not to model using a single internally layered element if shear stiffness is important.

The stress distribution through the wall section at 45 degrees from the $x_{1}$ axis under a shear load $Q_{1}=-1$ is shown for the $t / R=0.2$ tube section with the fiberglass-epoxy 
core and the balsa core in figure 10 and figure 11, respectively. The stresses are obtained using the highly discretized model (Model A) and the linear-cubic internally layered element model (Model C). Stresses are expressed in the laminate coordinate system. Stresses obtained using the highly discretized model are calculated using the constitutive relations and are shown at the Gauss point locations. For the internally layered element model, the in-plane and interlaminar stress distributions are obtained using the constitutive relations and the equilibrium equations, respectively.

It can be seen from figure 10(a) and figure 11(a) that the in-plane shear stress distribution $\tau_{13}$ obtained using the internally layered elements (Model C) are in good agreement with the Gauss point stresses of the detailed mesh (Model A) with a discrepancy of the maximum stress for the fiberglass-epoxy and balsa core of $0.2 \%$ and $1.6 \%$, respectively. It is seen in figure 10(b) and figure 11(b) that both models predict that the maximum interlaminar stress $\tau_{23}$ for the figerglass-epoxy core section occurs at the outer bonded-joint while the maximum for the balsa core section occurs at the inner bonded-joint. The discrepancy of the maximum stress for the fiberglass-epoxy and balsa core section is $7.5 \%$ and $12 \%$, respectively. The continuous interlaminar stresses and stress free surfaces is well captured by the equilibrium approach.

\subsection{Wind Turbine Blade Cross-Section}

This final example of the present paper concerns the analysis of the two-cell cross-section of a Siemens Wind Power A/S wind turbine blade shown in figure 12(a). The cross-section's shell and spar cap are made of fiberglass-epoxy, while the sandwich core present in the trailing edge walls and tail are made of balsa and foam, respectively. This section is located on the outboard section of the blade which is indicated by the thick spar cap. Figure 12(b) shows the mesh of the wind turbine cross-section obtained using a single layer of internally layered elements. The walls, web and spar cap are modelled using 28 16-node internally layered elements with cubic-linear interpolation in the cross-section plane and Hermitian cubic interpolation in the axial direction. The trailing core and transitions junctions are modelled using 17 8-node internally layered elements with linear interpolation in the cross-section plane and Hermitian cubic interpolation in the axial direction. The discretization contains a total of 45 elements. The position of the nodes depends only on the geometry of the section and is therefore independent of the material layup.

The location of the elastic center and the shear center are shown in figure 12(b) using a circle and a cross, respectively. The principal axes of bending and shear shown at the elastic center and shear center, respectively, are practically parallel. Furthermore, the axis associated with edgewise bending is almost aligned with the chord line of the section. The extension and bending stiffnesses about the principal axes of bending as well the torsion and shear stiffnesses about the principal axes of shear for the blade cross-section are presented in Table 2. The stiffness properties indicate that the bending stiffness in the edgewise direction is about four

Table 2: Stiffness properties of the blade section.

\begin{tabular}{cccccc}
\hline$E A[\mathrm{~N}]$ & $E I_{1}\left[\mathrm{Nm}^{2}\right]$ & $E I_{2}\left[\mathrm{Nm}^{2}\right]$ & $G J\left[\mathrm{Nm}^{2}\right]$ & $G A_{1}[\mathrm{~N}]$ & $G A_{2}[\mathrm{~N}]$ \\
\hline $2.22 \mathrm{E}+09$ & $2.28 \mathrm{E}+07$ & $9.40 \mathrm{E}+07$ & $3.24 \mathrm{E}+06$ & $1.65 \mathrm{E}+08$ & $2.50 \mathrm{E}+07$ \\
\hline
\end{tabular}



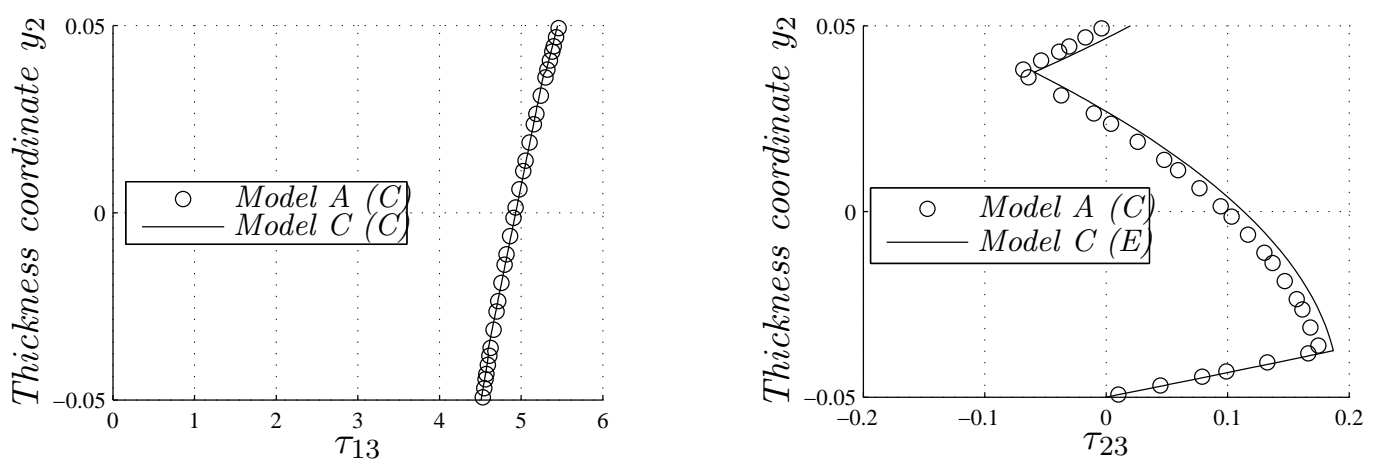

Figure 10: Fiberglass-epoxy core: (a) Constitutive derived (C) in-plane shear stresses $\boldsymbol{\tau}_{\mathbf{1 3}}$, (b) Constitutive derived (C) and equilibrium derived (E) transverse shear stresses $\boldsymbol{\tau}_{\mathbf{2 3}}$.
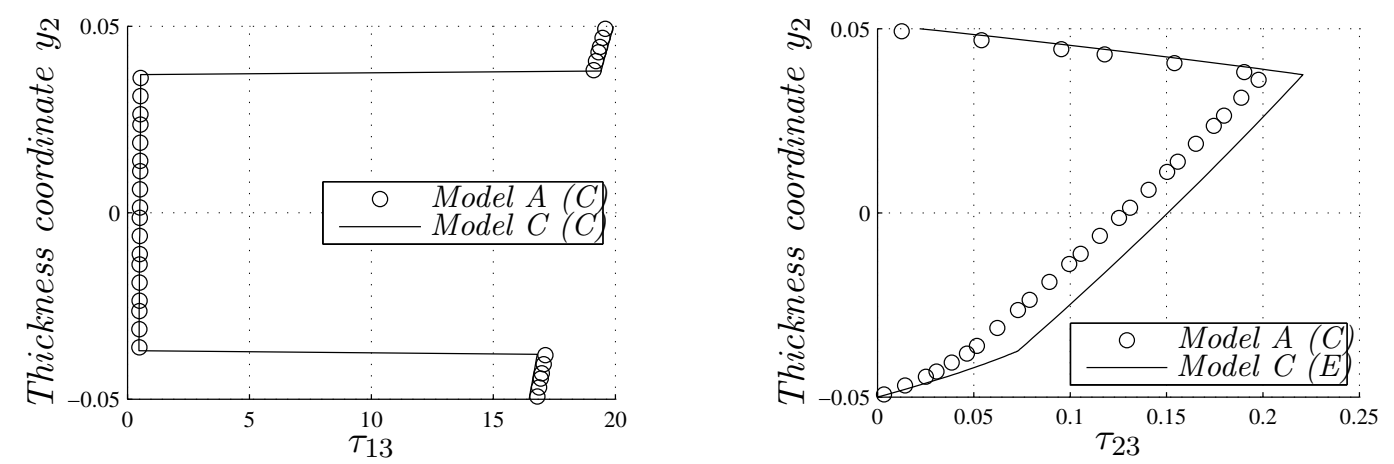

Figure 11: Balsa core: (a) Constitutive (C) in-plane shear stresses $\tau_{13}$, (b) Constitutive (C) and equilibrium (E) transverse shear stresses $\tau_{23}$.

times as large as the bending stiffness in the flapwise direction. The shear stiffness in the edgewise direction is about seven time as large as the shear stiffness in the flapwise direction which agrees with the results in Høgsberg and Krenk [7] where a detailed stress distribution shows the dominating effect of the spar cap in the edgewise shear stiffness. The shear center is almost located at the quarter-chord point which means that little twist will be induced from the lift distribution at a cross-sectional level.

Figure 13 shows the stress distribution at mid-height of the web for two pure shear load cases with $Q_{1}=1$ and $Q_{2}=1$, respectively, and one pure torsion load case with $M_{3}=1$. The loads are applied in the principal axes of shear. The thickness coordinate $\eta$ is positive towards the leading edge. The in-plane stress distribution $\tau_{13}$ shown in figure 13(a) is obtained using the constitutive relation, while the transverse interlaminar stress distribution $\tau_{23}$ shown in figure 13(b) is obtained using the stress recovery method. The stresses are normalized with respect to the largest shear stress of the pure shear load case with $Q_{2}=1$.

The sandwich construction of the web is apparent from the in-plane stress distribution $\tau_{13}$ shown in figure 13(a) where it can be seen that the faces carry most of the shear flow, while the soft core has little contribution in all three load cases. The largest in-plane stress $\tau_{13}$ occurs in the pure shear load case with $Q_{2}=1$ which confirms engineering intuition that the web has the largest influence on the flapwise shear stiffness $G A_{2}$. The largest in-plane stress 

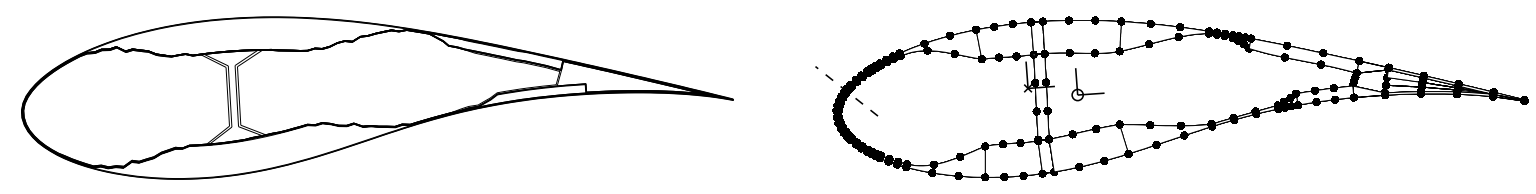

Figure 12: (a) Schematic of rotor blade cross-section (b) Discretization using 45 internally layered elements.

in the other shear load case and torsion load case are $28 \%$ and $1 \%$ of the largest stress of the load case with $Q_{2}=1$, respectively. The transverse shear stresses $\tau_{23}$ shown in figure 13(b) are three orders of magnitude smaller than the in-plane stresses $\tau_{13}$. The pure shear load case with $Q_{1}=1$ has interlaminar stresses $\tau_{23}$ with larger magnitude and opposite direction than those of the other shear load case with $Q_{2}=1$ even though the former's in-plane stresses are smaller and in the same direction. From (17) this indicates that for the shear load case with $Q_{1}=1$ the stress gradients $\frac{\partial \tau_{13}}{\partial y_{1}}$ and $\frac{\partial \sigma_{3}}{\partial y_{3}}$ in the web are larger and with opposite directions compared to the shear load case with $Q_{2}=1$.

The stress distribution through the leading edge wall section under an axial force $Q_{3}$ applied at the elastic center is shown in figure 14. The location of the section is indicated by the dashed line in figure 12(b). The axial stress distribution $\sigma_{3}$ shown in figure 14(a) is obtained using the constitutive relation, while the transverse interlaminar stress distribution $\sigma_{2}$ shown in figure 14(b) is obtained using the stress recovery method. Stresses are expressed in the laminate coordinate system and normalized with respect to the maximum axial stress $\sigma_{3}$.

The variation in axial stress distribution $\sigma_{3}$ shown in figure 14(a) comes from the different fiber orientation of each layer. The inner layer carries most of the axial load since it had the largest proportion of longitudinal fibers. Although only loaded axially, transverse stresses are present from varying in-plane contraction from dissimilar Poissons's ratio between the curved layers. This interlaminar stress is dominated by the curvature in the wall which corresponds to the last term in (19) indication that the magnitude of the transverse stresses will grown proportional with the wall curvature. The stress recovery indicates that the lower part of the wall experiences transverse compression, while the largest tensile transverse stress occurs at the bonded-joint between the middle and inner laminas. The largest transverse stress $\sigma_{2}$ is three orders of magnitude smaller than the maximum axial stress $\sigma_{3}$. It is noted that transverse tensile strength is typically two orders of magnitude smaller than the longitudinal tensile strength. The stress recovery method captures the interlaminar stress continuity between the layers and the stress free surfaces.

\section{Conclusion}

A previously developed theory for cross-sectional analysis has been extended using finite elements which can accommodate several laminas in one element and is supplemented by a technique to accurately determine the interlaminar stress distribution. In the approach, the in-plane stress distribution is obtained using the constitutive relation, while the transverse stress distribution is recovered by postprocessing the in-plane stress gradients via equilibrium equations of 3D elasticity derived in the laminate coordinate system. The method shows good agreement with a conventional highly discretized model, illustrated by a composite tube cross- 

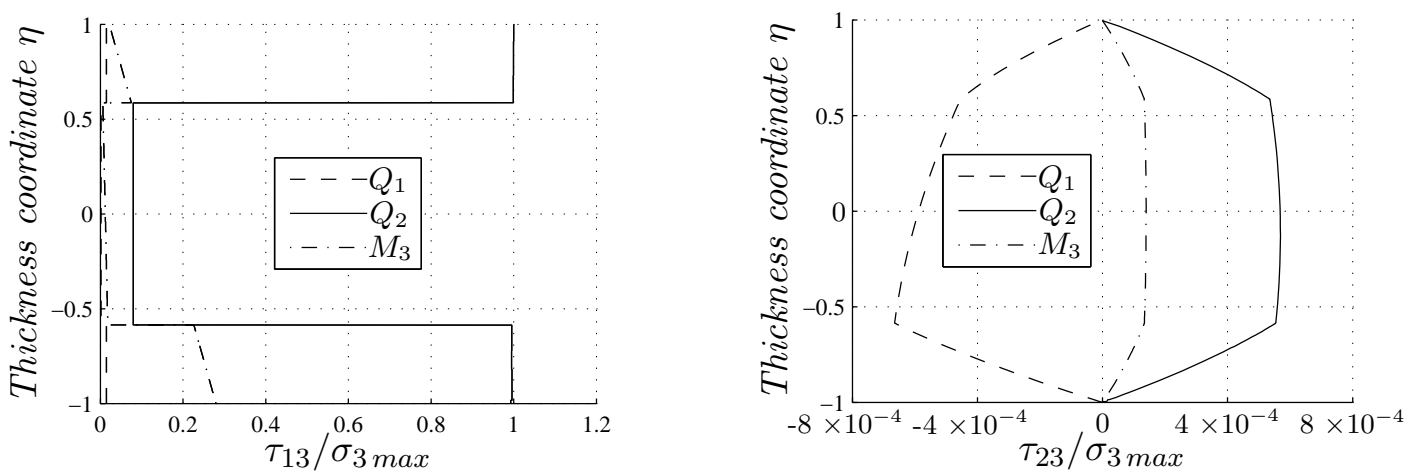

Figure 13: (a) Normalized constitutive in-plane shear stresses $\tau_{13}$, (b) Normalized equilibrium transverse shear stresses $\tau_{23}$.
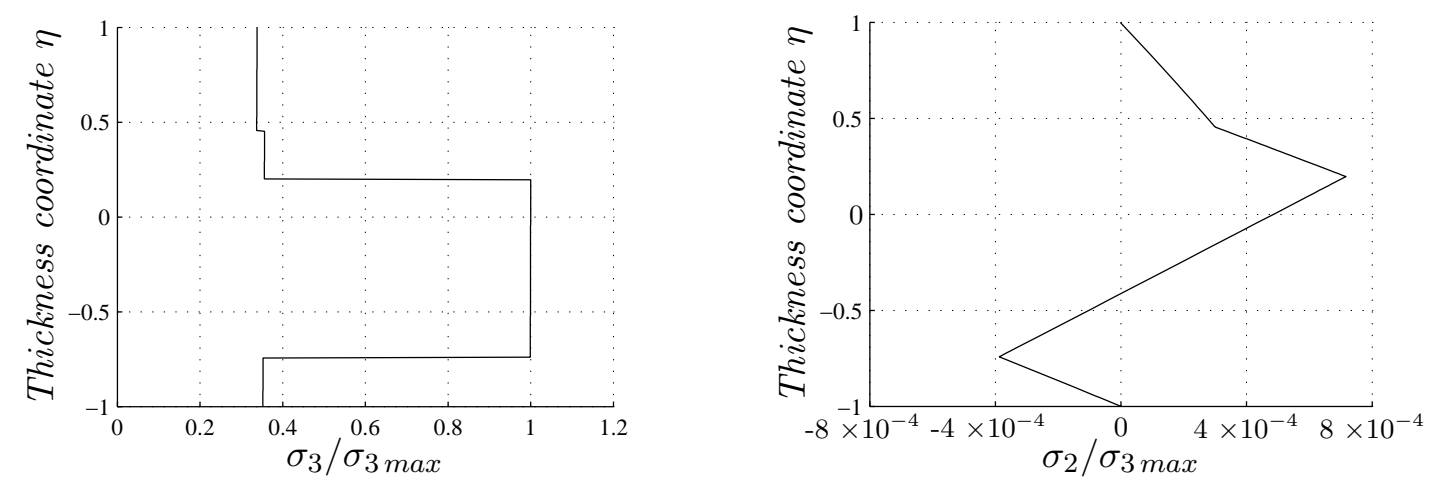

Figure 14: (a) Normalized constitutive in-plane axial stresses $\sigma_{3}$, (b) Normalized equilibrium transverse interlaminar stresses $\sigma_{2}$.

section with soft and stiff core. The results highlight that the use of internally layered elements for cross-section analysis can significantly reduce mesh generation effort and computational time while maintaining accuracy up to moderately thick walls. Postprocessing of stresses of a wind turbine cross-section showed the presence of transverse interlaminar stresses from in-plane contraction associated with varying Poisson's ratio between layers. Although the present paper deals only specifically with the stiffness properties, the present approach can be easily extended to provide the general cross-section mass properties.

\section{Acknowledgments}

This work has been supported by Siemens Wind Power A/S.

\section{References}

[1] Giavotto, V., Borri, M., Mantegazza, P., Ghiringhelli G., Carmaschi V., Maffioli, G.C., and Mussi, F., "Anisotropic Beam Theory and Applications", Computers and Structures, Vol. 16, No. 1-4, 1983, pp. 403-413. 
[2] Ghiringhelli, G.L., and Mantegazza, P., "Linear, Straight and Untwisted Anisotropic Beam Section Properties from Solid Finite Elements", Composites Engineering, Vol. 4, No. 12, 1994, pp. 1225-1239.

[3] Cesnik, C. E., and Hodges, D. H., "VABS: A New Concept for Composite Rotor Blade Cross-Sectional Modeling", Journal of the American Helicopter Society, Vol. 42, No. 1, 1997, pp. 27-38.

[4] Hodges, D. H., and Yu, W., "A Rigorous, Engineer Friendly Approach for Modelling Realistic, Composite Rotor Blades," Wind Energy, Vol. 10, 2007, pp. 179-193.

[5] Couturier, P. J., Krenk, S., and Høgsberg, J., "Beam Section Stiffness Properties using a Single Layer of 3D Solid Elements", Computers and Structures, Vol. 156, 2015, pp.122133.

[6] Chen, H., Yu, W., and Capellaro, M., "A Critical Assessment of Computer Tools for Calculating Composite Wind Turbine Blade Properties", Wind Energy, Vol. 13, 2010, pp. 497-516.

[7] Høgsberg, J., Krenk, S., "Analysis of Moderately Thin-Walled Beam Cross Sections by Cubic Isoparametric Elements", Computers and Structures, Vol. 134, 2014, pp. 88-101.

[8] Márquez, C. G., "New Layered Finite Element Formulations for the BEam Cross Section Analysis Software", M.Sc. Thesis, Wind Energy Dept., Technical University of Denmark, Lyngby, Denmark, 2013.

[9] Wang, A. S. D., and Crossman, F. W., "Calculation of Edge Stresses in Multi-Layer Laminates by Sub-Structuring", Journal of Composite Materials, Vol. 12, 1978, pp. 7683.

[10] Panda, S. C., and Natarajan, R., "Finite element analysis of laminated composite plates", International Journal for Numerical Methods in Engineering, Vol. 14, No. 1, 1979, pp. 6979.

[11] Jones, R., Callinan, R., Teh, K. K., and Brown, K. C., "Analysis of Multi-Layer Laminates using Three-Dimensional Super-Elements", International Journal for Numerical Methods in Engineering, Vol. 20, No. 3, 1984, pp. 583-587.

[12] Chang, F. K., Perez, J. L., and Chang, K. Y., "Analysis of Thick Laminated Composites", Journal of Composite Materials, Vol. 24, 1990, pp.801-821.

[13] Krenk, S., "Element Stiffness Matrix for Beams with General Cross-Section Properties," Report, Technical University of Denmark, Department of Mechanical Engineering, Denmark, 2006.

[14] Couturier, P., and Krenk, S. "Composite Beam Cross-Section Analysis by a Single HighOrder Element Layer," Proceedings of the 56th Structures, Structural Dynamics, and Materials Conference, AIAA, Reston, Virginia, January, 2015.

[15] Couturier, P., and Krenk, S. "General Beam Cross-Section Analysis using a 3D Finite Element Slice," Proceedings of the ASME 2014 International Mechanical Engineering Congress and Exposition, ASME, November, 2014. 
[16] Cook, R. D., Malkus, D. S., Pleasha, M. E., Witt, R. J., Concepts and Applications of Finite Element Analysis, 4th ed., Wiley, New York, 2002.

[17] Bathe, K. J., Finite Element Procedures, Prentice-Hall, Englewood Cliffs, N.J., 1996.

[18] Hughes, T. J. R., The Finite Element Method, Prentice Hall, Englewood Cliffs, N.J., 1987.

[19] Griffin, D. A., "WindPACT Turbine Design Scaling Studies. Technical Area 1 - Composite Blades for 80- to 120- Meter Rotor", National Renewable Energy Laboratory, Golden, CO, NREL/SR-500-29492, 2001.

[20] Steinboeck, A., Kugi, A., and Mang, H. A., "Energy-Consistent Shear Coefficients for Beams with Circular Cross Sections and Radially Inhomogeneous Materials", International Journal of Solids and Structures, Vol. 50, No. 11, 2013, pp. 1859-1868. 